\title{
Enterocytozoon bieneusi in AIDS: symptomatic relief and parasite changes after furazolidone
}

D Dionisio, L Ibba Manneschi, S Di Lollo, A Orsi, G Sterrantino, M Meli, M Gabbrielli, A Tani, A Papucci, F Leoncini

Abstract

Aims-To investigate changes in morphology of the developmental stages of Enterocytozoon bieneusi and symptomatic relief observed in AIDS patients after treatment with furazolidone.

Methods-Six AIDS patients with symptomatic $E$ bieneusi infection of the small intestine were treated with a course of furazolidone. All patients had a weekly monitoring of parasite shedding in stool by light microscopy during and after treatment. At the end of the treatment, duodenal biopsy specimens obtained from three patients were studied by transmission electron microscopy by two pathologists who were unaware of the patients' treatment.

Results-All patients showed both clinical and parasitological response with transient clearance or decrease of spore shedding in stool. After treatment, alterations in faecal spores were observed in all patients by light microscopy, and ultrastructural changes in $E$ bieneusi at all stages of the life cycle were demonstrated in biopsy specimens of the three patients who underwent post-treatment endoscopy.

Conclusions-The clinical benefit seen after treatment with furazolidone in six AIDS patients with $E$ bieneusi intestinal infection may be due to damage to the developmental stages causing a partial inhibition to reproduction of the parasite. $(\mathcal{F}$ Clin Pathol 1997;50:472-476)

Keywords: Enterocytozoon bieneusi; furazolidone; parasite morphology; AIDS

Enterocytozoon bieneusi is a microsporidian that causes chronic diarrhoea in severely immunocompromised patients with AIDS. ${ }^{1}$ Currently no established therapy is available because none of the available agents lead to eradication of the infection nor significantly affect the life cycle of the parasite; moreover, relapses rapidly occur after treatment is discontinued. ${ }^{1-4}$

Furazolidone is a synthetic nitrofuran that is active against a broad spectrum of Gram negative and Gram positive bacteria as well as against some intracellular or extracellular
Unit, Careggi Hospital,

Florence, Italy

D Dionisio

G Sterrantino

M Meli

F Leoncini

Department of Human Anatomy and

Histology, University

of Florence, Italy

L Ibba Manneschi

A Tani

Institute of Pathology, University of Florence, Italy

S Di Lollo

A Papucci

Laboratory for

Microbiology and

Virology, Careggi

Hospital, Florence,

Italy

A Orsi

Gastroenterology Unit,

Careggi Hospital,

Florence, Italy

M Gabbrielli

Correspondence to

Dr Dionisio, Infectious

Diseases Unit, Careggi

Hospital, V. le Morgagni 85

50139, Florence, Italy.

Accepted for publication 25 February 1997

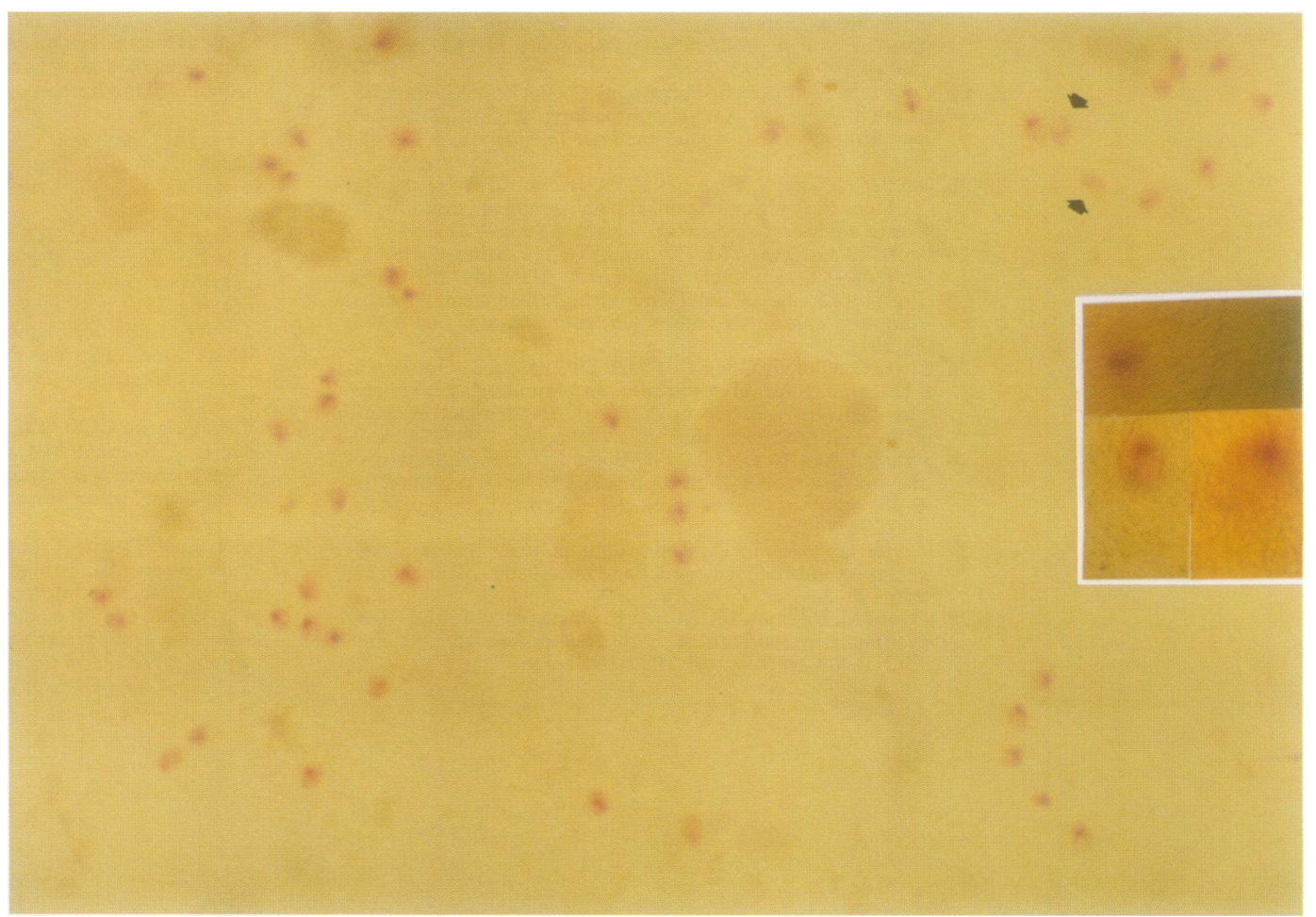

Figure 1 Microsporidian spores (stool specimen after treatment). Many spores are present, almost all showing a marked red stained clot. Normal spores are sporadically seen (arrows) (original magnification $\times 1000)$. Inset: Changed spores with evidence of the inner red stained clot (original magnification $\times 1000$ ). 


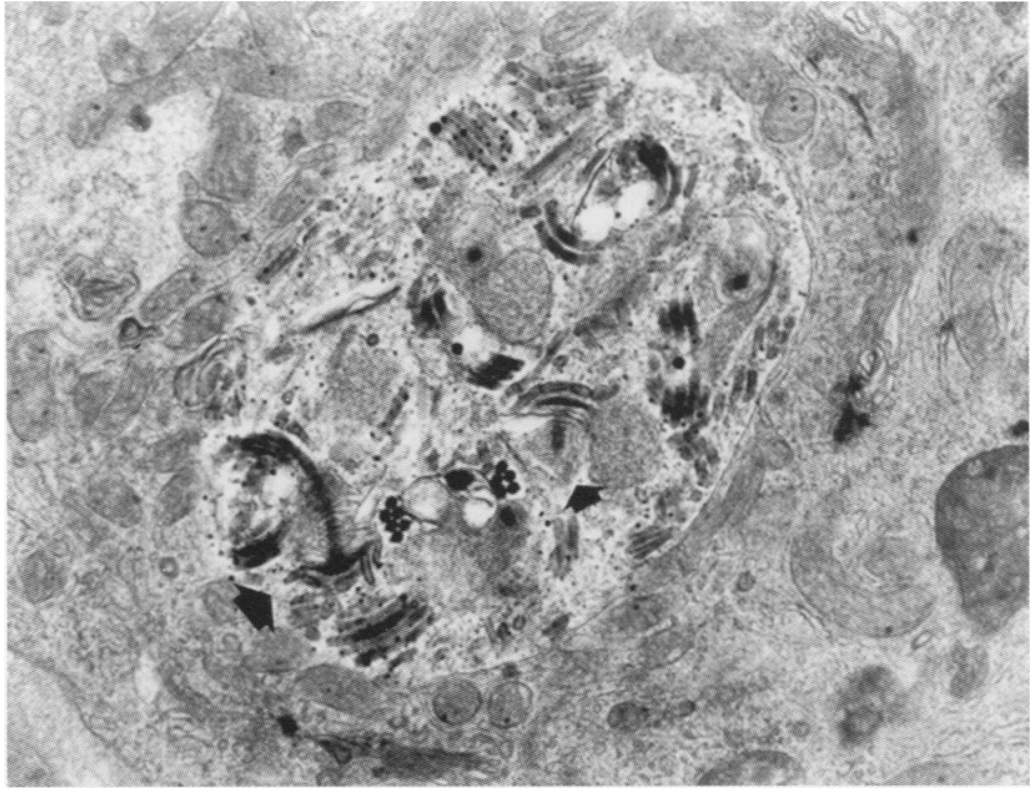

Figure 2 Normal late sporogonial plasmodium (biopsy specimen before treatment). Dense nuclei are each associated with electron dense discs fused into arcs forming polar tube coils. Umbrella shaped anchoring discs and associated polaroplast membranes attached to the manubroid portion of a polar tube are present (arrows). Electron lucent inclusions are seen in elongate and cross section (original magnification $\times 9000$ ).

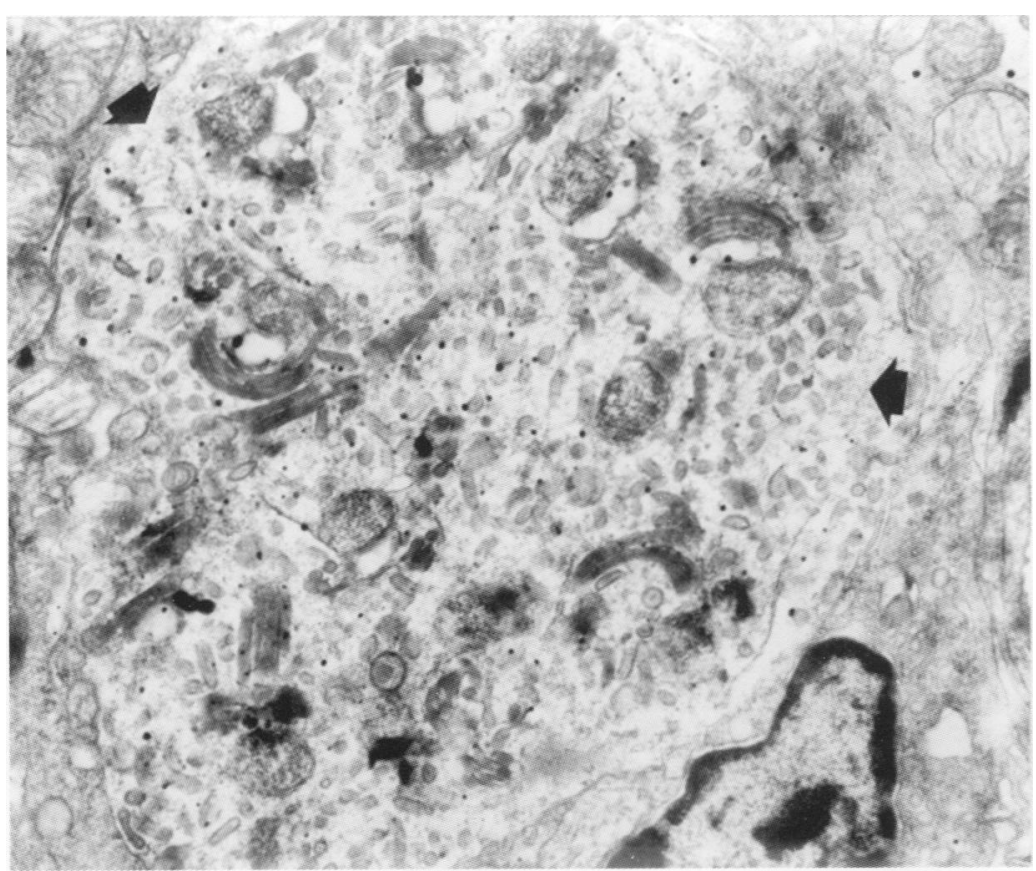

Figure 3 Late sporogonial plasmodium (biopsy specimen after treatment). The stage of development is similar to that in figure 2. Many nuclei are present but vacuolated due to irregular distribution of chromatin. Some polar tube coils are evident but most of the electron dense discs fail to develop into polar tubes and appear disorganised, disrupted, and pale (arrows) (original magnification $\times 8000$ ).

protozoa (trichomonas, giardia, entamoeba, blastocystis, leishmania)..$^{5-8}$ One report about its effectiveness against Isospora belli has also been published. ${ }^{9}$ Activity of furazolidone results from its ability to inhibit various enzymes of the Krebs cycle, namely those involved in the acetyl-CoA synthesis from piruvate. ${ }^{10}$ Following oral administration the drug is well absorbed and achieves therapeutic concentrations in blood and significant concentrations in bile and urine. ${ }^{11}$ It has a good safety profile and does not alter the normal intestinal flora. ${ }^{10}$ In a preliminary study in three AIDS patients the drug induced remission of $E$ bieneusi infection symptoms as well as transient stopping of spore shedding in stool. ${ }^{12}$

This study aimed to confirm the clinical benefit of furazolidone and to describe alterations of parasite morphology in stool and in duodenal biopsy specimens after treatment.

\section{Methods}

Six HIV infected men with symptomatic $E$ bieneusi infection were studied, including the three patients from the preliminary work. ${ }^{12}$ The patients' ages ranged between 26 and 36 years (mean 30.6). Three patients were drug addicts, two were homosexuals, and one was haemophiliac. $\mathrm{CD}^{+} \mathrm{T}$ cell counts ranged between 8 and $46 \times 10^{6} / 1$ (mean 24). All six patients had AIDS at the time of study entry; AIDS defining events were oesophageal candidiasis in three cases, cryptococcosis in two cases, and Kaposi's sarcoma in one case. All patients had chronic diarrhoea (at least two months) with one to six watery stools per day. Weight loss ranged between 3 and $10 \mathrm{~kg}$ (mean 5.5).

Stool were processed according to the method of Weber et $a l^{13}$ and Van Gool et al ${ }^{14}$ to detect microsporidia. The amount of spore shedding was evaluated by a semiquantitative criterion on 100 microscopic fields: spores were defined as very frequent if present in every field, frequent if present in more than 50 but fewer than 100 fields, rare if present in fewer than 50 fields, and very rare if sporadically present. Before study entry, frequent to very frequent spores of microsporidia were present in stool of all patients. Stool samples were also examined for bacteria, Clostridium difficile toxin, mycobacteria, eggs and parasites, adenoviruses, and rotaviruses with negative results.

Transmission electron microscopy (TEM) was performed on endoscopic biopsy specimens taken from the third tract of duodenum, before treatment. At least two samples from each patient were processed. Specimens were fixed with glutaraldehyde $2.5 \%$ in cacodylate buffer ( $\mathrm{pH}$ 7.4) for three to four hours at room temperature, washed in the same buffer and postfixed in osmium tetroxide in $1 \%$ Milloning buffer (Sic, Rome, Italy) for one hour at room temperature. After dehydration through graded acetone series, the specimens passed through propylene oxide and were embedded in Epon 812. Semi-thin sections $(2 \mu \mathrm{m})$ were cut, stained with toluidine blue-Na tetraborate and studied under light microscope. About 100 ultrathin sections were obtained from all the selected specimens and were stained with uranyl acetate and alkaline bismuth subnitrate. For each section at least three random fields were examined by TEM. Care was taken to evaluate only areas away from the borders of the biopsy specimen to avoid traumatic artefact.

The haemophiliac patient denied consent for endoscopic biopsies, and TEM study of biopsy specimens was negative in one of the homosexual patients. In these two patients the diagnosis of $E$ bieneusi infection was achieved by a TEM study of stool samples collected before treatment. Stool specimens were fixed in $10 \%$ formalin, and excess faecal debris was removed 


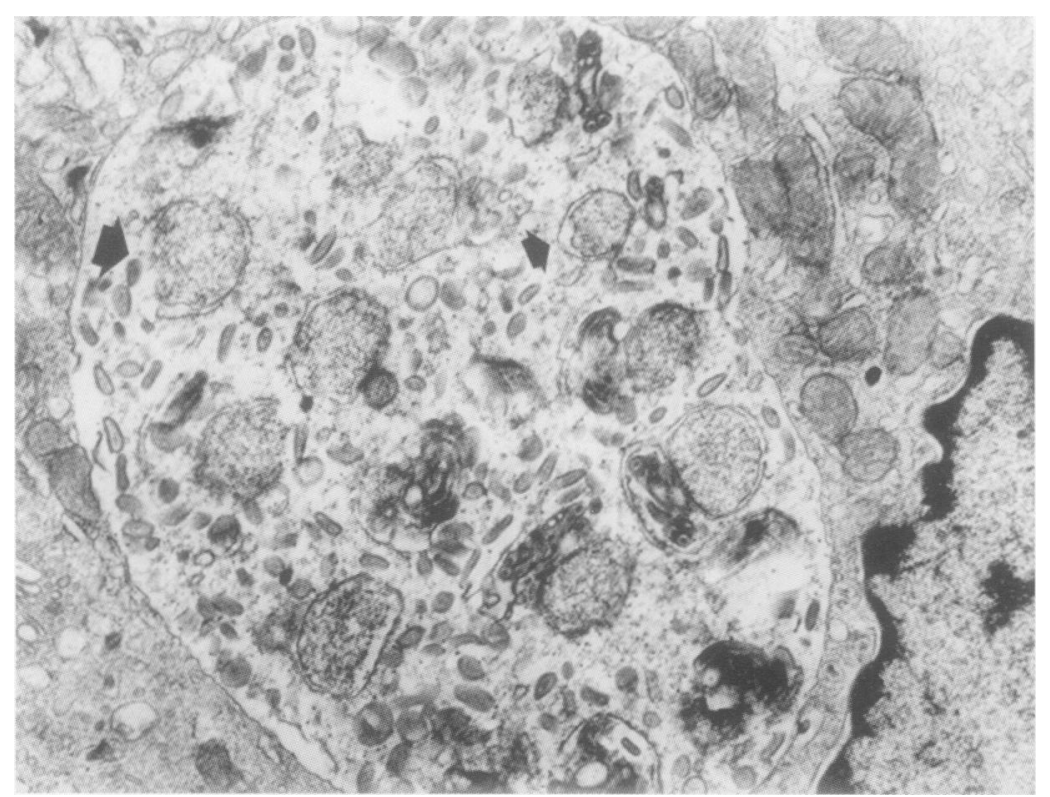

Figure 4 Sporogonial plasmodium (biopsy specimen after treatment). Many nuclei are evident but chromatin appears disarranged. Disruption of nuclear membranes and apparent separation of the nuclear membrane from nucleoplasm are seen (arrows). Some nuclei are irregular in outline. Electron dense discs appear disorganised and fail to fuse into arcs. Many of them are deformed (original magnification $\times 10000$ ).

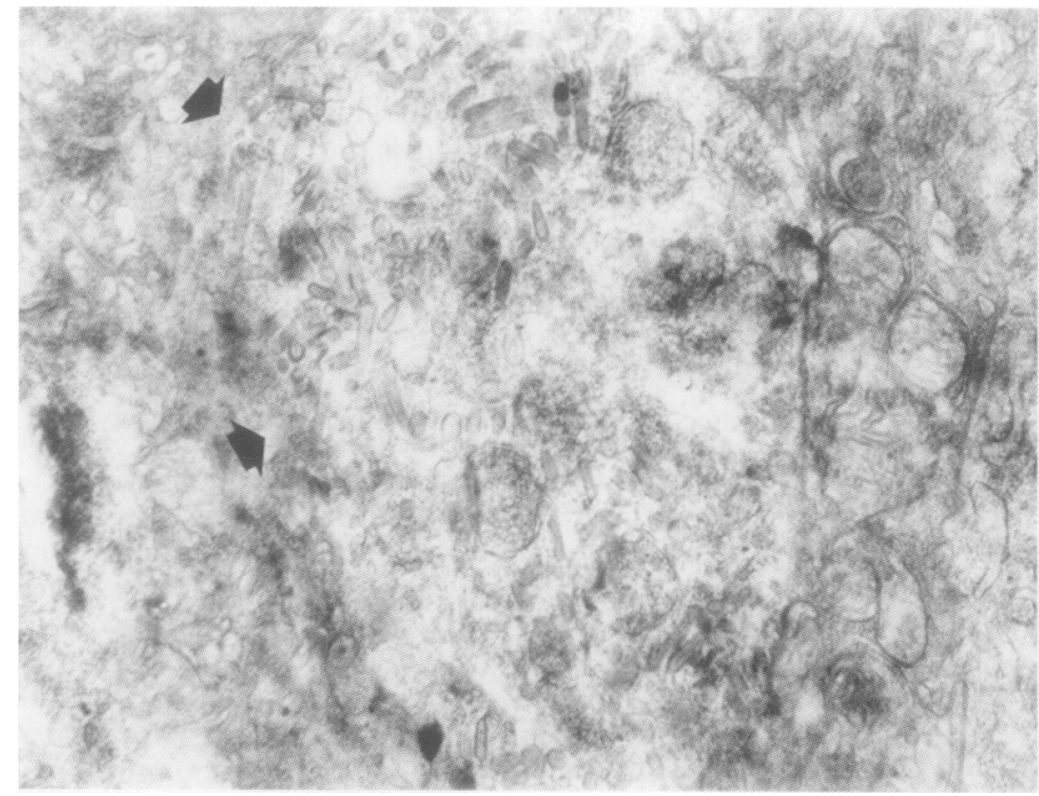

Figure 5 Sporogonial plasmodium (biopsy specimen after treatment). Nuclei are deformed with disarrangement of chromatin and disruption of nuclear membrane. Electron dense discs are extremely disorganised and pale and do not fuse into arcs (arrows) (original magnification $\times 11000$ ).
Furazolidone-N-(5-nitro-2-furfurilidene)-3 amino-2-oxazolidone- $100 \mathrm{mg}$ tablets, was given orally four times daily for 20 days to one group of three patients (cases 1-3). The remaining three patients (cases 4-6) were treated with furazolidone $100 \mathrm{mg}$ tablets five times daily for 18 days. Written informed consent was obtained before treatment. No concomitant antidiarrhoeal drugs were administered. Clinical assessment, and haematological and liver function tests were monitored after 10 days and at the end of the treatment. Adverse events to the drug were evaluated.

In all patients spore shedding in stool was monitored weekly during and after treatment with both Weber's and Van Gool's stains, and stool frequency and body weight were recorded.

The three patients treated with furazolidone for 18 days underwent upper intestinal endoscopy three days after completion of therapy. At least two biopsy specimens were taken from the third part of duodenum and processed as described above. Ultrathin sections were examined by TEM by two pathologists blind to the patients' treatment or clinical outcome. In the same patients a TEM study on stool samples collected during the follow up period was also done as described above.

\section{Results}

CLINICAL RESPONSE

In all patients there was remission of diarrhoea within two weeks of starting treatment, and clearance or great decrease of spore shedding in stool after treatment were observed. Neither side effects nor alterations in the laboratory parameters attributable to the drug were observed.

Case 1 showed persistent clinical remission over the two months following completion of treatment and body weight increase. Subsequently, relapse of diarrhoea with very frequent faecal spores required a new cycle with furazolidone $200 \mathrm{mg}$ three times daily for three days. Diarrhoea resolved within one day and reexamination of stool one week later demonstrated only very rare spores. Normal bowel movements with formed stool lasted until the patient died, 40 days later, having developed systemic cytomegalovirosis.

Case 2 had intermittent shedding of very rare spores in stool, but he had neither clinical nor parasitological evidence of reactivation until he died one month later from unexplained acute abdomen.

Case 3 remained asymptomatic with only very rare spores in stool until he died 15 days later having developed acute encephalopathy.

Case 4 was symptom-free and had no spores in stool during the following six weeks. Thereafter clinical and parasitological relapse occurred, and a new cycle with furazolidone was administered. The patient showed remission of symptoms within two weeks of starting treatment, and transient clearance of spore shedding in stool after therapy. Subsequently, although intermittent shedding of rare to very rare spores was documented in stool, he had no
All patients underwent a baseline laboratory evaluation that included full blood cell count, urine analysis, electrolytes, and liver function tests. 


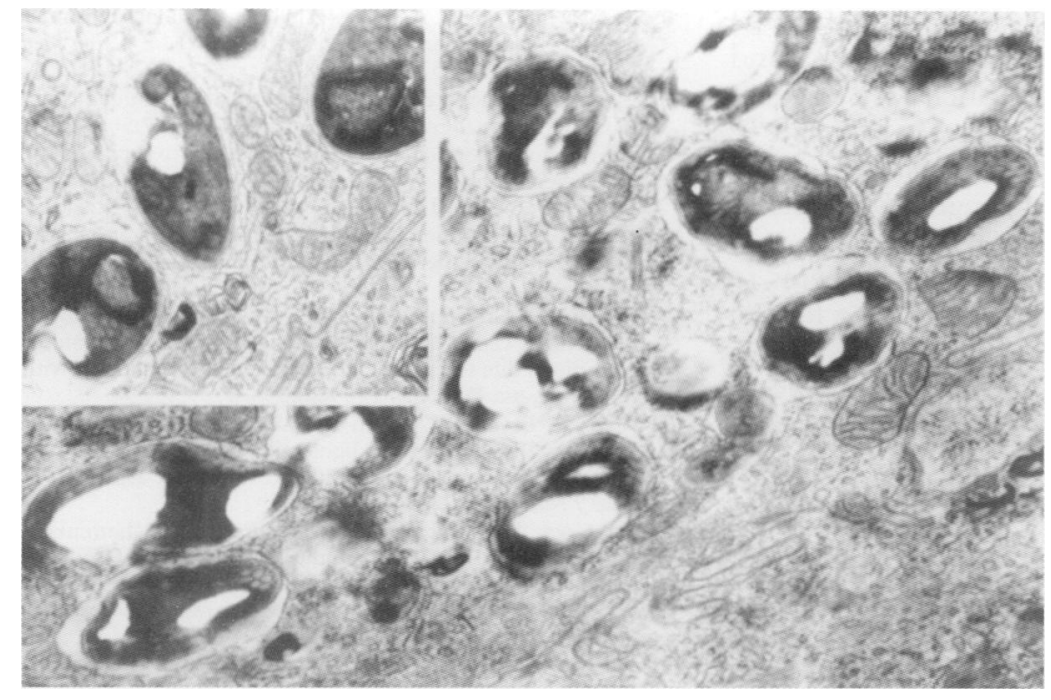

Figure 6 Mature spores (biopsy specimen after treatment). The spores show a wavy outline but polar tubes are normal. Diastasis is seen between each spore and the enterocyte cytoplasm (original magnification $\times 10000$ ). Inset: Normal mature spores in a biopsy specimen before treatment (original magnification $\times 10$ 000).

diarrhoea over the following four months until he died from systemic Mycobacterium avium infection.

Case 5 was asymptomatic, even though very rare spores were intermittently documented in stool, until he died 11 weeks later having developed systemic cryptococcosis.

Case 6 had long lasting clinical remission and body weight increase. Although rare to very rare spores were occasionally detected in stool, this patient was free from symptoms four months after completion of treatment and had gained $3 \mathrm{~kg}$ in weight.

PARASITE MORPHOLOGY IN STOOL

Light microscopy

In all patients alterations in faecal spores were detected by Weber's method after treatment. In these cases the pinkish-red belt-like stripe as originally described by Weber et $a l^{13}$ was replaced by a marked red stained clot (fig 1); however, spore shape and size was normal. The number of dysmorphous spores was variable, ranging from 1 to 50 in each microscopic field (mean 10). Changed spores were intermittently excreted, and coexisted with normal spores with a mean ratio of $5: 1$. Alterations in faecal spores were not demonstrated by Van Gool's method.

\section{Transmission electron microscopy}

TEM study of stool samples collected after therapy demonstrated $E$ bieneusi spores with normal polar tubes but a wavy wall outline.

\section{PARASITE MORPHOLOGY IN BIOPSY SPECIMENS} Only normal stages of $E$ bieneusi ${ }^{15}$ were observed in all studied ultramicroscopic fields from the biopsies taken before treatment (fig 2). After treatment parasite abnormalities at all stages of the life cycle were documented in biopsies from the three studied patients and appeared most pronounced in parasites undergoing their sporogony phases. In these cases disarrangement of nuclei chromatin was constantly present (figs 3-5), disruption of nuclear membrane, irregularity of nuclear outline, and apparent separation of nuclear membrane from the nucleoplasm were also frequently seen (figs 4 and 5).

Moreover, about half of sporogonial plasmodia seen in the examined fields showed electron dense discs (EDDS) that failed to develop into polar tubes and appeared disorganised, deformed, disrupted, and pale (figs 3-5). Sporoblasts showed disarrangement of nuclear chromatin similar to that observed in sporogonial plasmodia. Most spores had an irregular outline with diastasis between the spore and the enterocyte cytoplasm suggesting shrinking; however, the polar tubes were unchanged (fig 6).

No alterations in host cell structures were observed in biopsy specimens taken before and after treatment.

\section{Discussion}

Furazolidone treatment in six patients with $E$ bieneusi intestinal infection led to symptomatic relief and decrease (or transient absence) of spore shedding in stool. Subsequently, two patients showed clinical and parasitological relapse during the second and third month following discontinuation of therapy; however, clinical and parasitological remission was achieved by a new course of treatment.

Biopsy specimens from the three patients who underwent post-treatment endoscopy showed ultrastructural changes in $E$ bieneusi at all stages of the life cycle, although these were more pronounced for sporogonial plasmodia whose nuclei constantly showed alterations. Many sporogonial plasmodia showed severe changes in EDDS, and most spores showed a distortion in their outline that suggests shrinking.

Although abnormalities of nuclei have been described in $E$ bieneusi after albendazole treatment, ${ }^{3}$ to our knowledge marked changes in EDDS have not been reported previously. EDDS are essential to the harmful potential of microsporidia, being precursors of polar tubes. ${ }^{15}$ Therefore, the impaired development of EDDS found in sporogonial plasmodia probably indicates a proportionally impaired offending activity of $E$ bieneusi. Distortion in spore outline observed in biopsy samples is difficult to explain; however, we can exclude that it was an artefact, as cell organelles surrounding the spores were normal.

The alterations in developmental stages of $E$ bieneusi seen in biopsy specimens by TEM can justify the presence of dysmorphous spores in stool in all patients. To our knowledge, these changes in faecal spores observed by Weber's method after furazolidone treatment have not been reported previously after any other treatment for $E$ bieneusi, ${ }^{1}{ }^{13}{ }^{16-18}$ and were not seen in our patients before treatment. Alterations in faecal spores were not demonstrated by Van Gool's method, as the fluorochrome employed in this method does not penetrate spores, but only binds to chitin, a component of the microsporidian spore wall. ${ }^{14}$

The electron microscopic alterations observed on biopsies taken after treatment suggest that furazolidone has a direct activity on $E$ bieneusi, likely by inhibiting parasite mul- 
tiplication. This hypothesis is supported by the observed decrease of parasite load in stool and by the subsequent symptomatic relief. Furazolidone, however, was not able to eradicate $E$ bieneusi infection as, in spite of treatment, some parasites accomplished normal development, giving rise to normal spores.

In spite of its small size, this study seems to demonstrate the efficacy of a new drug for the treatment of $E$ bieneusi intestinal microsporidiosis. The observed symptomatic relief due to furazolidone strictly correlated with the morphological changes of the life cycle of the parasite.

1 Bryan RT. Microsporidia. In: Mandell GL, Bennett JE, Dolin R, eds. Principles and practice of infectious diseases. New York: Churchill Livingstone 1995:2513-24.

2 Blanshard C, Ellis DS, Tovey DG, Dowell S, Gazzard BG. Treatment of intestinal microsporidiosis with albendazole in patients with AIDS. AIDS 1992;6:311-13.

3 Blanshard C, Ellis DS, Dowell SP, Tovey G, Gazzard BG. Electron microscopic changes in Enterocytozoon bieneusi following treatment with albendazole. $f$ Clin Pathol following treatment

4 Dieterich DT, Lew EA, Kotler DP, Poles MA, Orenstein JM. Treatment with albendazole for intestinal disease due to Enterocytozoon bieneusi in patients with AIDS. $\mathcal{F}$ Infect Dis 1994;169:178-83.

5 Prado Camacho JL. A comparison of furazolidone and ampicillin in the treatment of invasive diarrhoea. Scand 7 Gastroenterol 1989;24(suppl 169):54-9.

6 Shepherd RW, Boreham PFL. Recent advances in the diagnosis and management of giardiasis. Scand 7 Gastroenterol 1989;24(suppl 169):60-4.

7 Zierdt CH. Blastocystis hominis. Past and future. Clin Microbiol Rev 1991;4:61-79.
8 Berman JD, Lee LS. Activity of oral drugs against Leishmania tropica in human macrophages in vitro. $A m \mathcal{F}$ Trop $\mathrm{Med}$ Hyg 1983;32:947-51

9 Wittner M, Tanowitz HB, Weiss LM. Parasitic infections in AIDS patients. Infect Dis Clin North Am 1993;7:569-86.

10 Altamirano A, Bondani A. Adverse reactions to furazolidone and other drugs. A comparative review. Scand $\mathcal{f}$ Gastroen terol 1989;24(suppl 169):70-80.

11 White AH. Absorption, distribution, metabolism and excretion of furazolidone. Scand $\mathcal{F}$ Gastroenterol 1989;24(suppl 169):4-10.

12 Dionisio D, Sterrantino G, Meli M, Trotta M, Milo D, Leoncini F. Use of furazolidone for the treatment of microsporidiosis due to Enterocytozoon bieneusi in patients with AIDS. Recenti Prog Med 1995;86:394-7.

13 Weber R, Bryan RT, Owen RL, Wilcox CM, Gorelkin I Visvesvara GL. Improved light microscopical detection of microsporidia spores in stools and duodenal aspirates. $N$ Engl f Med 1992;326:161-6.

14 Van Gool T, Snijders F, Reiss P, Eeftinck Schattenkerk JKM, van den Bergh Weerman MA, Bartelsman JFWM, et al. Diagnosis of intestinal and disseminated microsporidial infections in patients with HIV by a new rapid fluorescence technique. F Clin Pathol 1993;46:694-9.

15 Cali A, Owen RL. Intracellular development of Enterocytozoon, a unique microsporidian found in the intestine of AIDS patients. F Protozool 1990;37:145-55.

16 DeGirolami PC, Ezratty CR, Desai G, McCullough A Asmuth D, Wanke $\mathrm{C}$, et al. Diagnosis of intestinal microsporidiosis by examination of stool and duodenal aspirate with Weber's modified trichrome and Uvitex $2 \mathrm{~B}$ stains. f Clin Microbiol 1995;33:805-10.

17 Didier E, Orenstein JM, Aldras A, Bertucci D, Rogers LB, Janney FA. Comparison of three staining methods for detecting microsporidia in fluids. 7 Clin Microbiol 1995;33. 3138-45.

18 Carter PL, MacPherson DW, McKenzie RA. Modified technique to recover microsporidian spores in sodium acetate-acetic acid-formalin-fixed fecal samples by ligh microscopy and correlation with transmission elect microscopy. f Clin Microbiol 1996;34:2670-3. 